\title{
Measuring the Efficiency of Interatomic Coulombic Decay in Ne Clusters
}

\author{
M. Förstel ${ }^{\mathrm{a}}$, T. Arion ${ }^{\mathrm{b}, \mathrm{c}}$, U. Hergenhahn ${ }^{\mathrm{a}}$ \\ ${ }^{a}$ Max-Planck-Institut für Plasmaphysik, EURATOM Association, Wendelsteinstr. 1, \\ 17491 Greifswald, Germany \\ ${ }^{b}$ Max-Planck-Institut für Plasmaphysik, EURATOM Association, Boltzmanstr. 2, 85748 \\ Garching, Germany \\ ${ }^{c}$ Present address: University of Hamburg, CFEL, Luruper Chaussee 149, 22761 \\ Hamburg, Germany
}

\begin{abstract}
The efficiency of interatomic coulombic decay (ICD) in Neon clusters with a mean size of $\langle N\rangle=480$ is measured directly. By detecting the photoelectrons and the ICD electrons in coincidence and normalizing their ratio using the detection probability of the respective electrons we show that the relaxation of Ne $2 s$ vacancies in Ne clusters by ICD has an efficiency of unity. Keywords: Interatomic Coulombic Decay, ICD, Clusters, Autoionization, Electron-Electron-Coincidence
\end{abstract}

\section{1. Introduction}

Core holes in atoms, molecules and bulk matter are known to decay almost exclusively by autoionization, called 'Auger decay' when autoionization

4 of a positively charged state is considered. For more shallow inner valence 5 holes Auger decay is energetically forbidden. In an aggregate they can nev-

Email addresses: marko.foerstel@ipp.mpg.de (M. Förstel), uwe.hergenhahn@ipp.mpg.de (U. Hergenhahn) 
ertheless autoionize, when the chemical environment of the initial vacancy assists by delocalizing the final state charge. The direct autoionization of an inner valence hole into a delocalized final state has been termed Interatomic Coulombic Decay (ICD) [1]. After its first experimental demonstration [2] this process has attracted considerable interest in the last decade (see e.g. the review papers $[3,4])$. As ICD has been shown to proceed on a femtosecond timescale [5-7], it seems highly plausible that it forecloses other relaxation channels, such as fluorescence or nuclear dynamics[8]. Often, it is tacitly assumed that such channels are completely quenched. It thus may seem surprising that few experiments have been documented, in which the efficiency of ICD was determined quantitatively and taking into account the branching ratios also into conceivable, non-autoionizing relaxation pathways. In our earlier work, we have shown for Ne clusters that the intensity of the $2 s$ photoelectron line, creating the initial state of ICD, equals the intensity of ICD electrons over a wide interval of cluster sizes [9]. We are not aware of other studies of this type however. This might be explicable because, despite its conceptual simplicity, performing an actual experiment on the competition of ICD with other mechanisms faces some difficulties. Thus there is a clear need for an experimental method to determine the efficiency of ICD in a quantitative and general way. Despite the fact that numerous studies have shown that ICD is a very important channel of electronic relaxation, its effectiveness has never been quantified.

In this contribution we demonstrate that analysis of photoelectron-ICD electron coincidence spectra can yield an accurate figure for the branching ratio of the ICD channel. By branching ratio we mean the percentage of Ne2s 
vacancies which decay via ICD. We show that relaxation of $2 s$ vacancies in Ne clusters by ICD has an efficiency of unity. Different than the study of Barth et al. [9], our experiment does not rely on a comparison between two features which are measured independently in the same spectrometer, but probes the electron pairs, which are causally correlated by ICD.

Why has no other experiment addressed this topic earlier? A technique which has been widely used to detect the signature of ICD is cold target recoil ion momentum spectroscopy (COLTRIMS, see [10]). While COLTRIMS is extremely powerful as it detects the occurence of ICD from the back-to-back Coulomb explosion of the final state (see e.g. [11] and examples cited in [4]), the same fact makes it 'blind' against other, non-autoionizing channels, in which no second positively charged fragment is produced. For the case of $\mathrm{Ne}$ clusters, we show here that such channels are below the $10 \%$ level, which is the sensitivity of our experiment.

We note that the situation is different for the ICD-like decay of coreexcited or core-ionized states [12]. Here, the main competing channel is Auger decay (decay into local two-hole final states), and a direct comparison between ICD and Auger final states is often possible, as they can be separated spectroscopically. If Auger decay and ICD are both energetically allowed, as a rule of thumb Auger decay is the more important channel.

\section{Experimental}

Interatomic or Intermolecular Coulombic Decay can be initiated by different excitation mechanisms. For the sake of its study, photoionization by synchrotron radiation offers the advantage of a controlled energy deposition 
in the initial state, which then decays. Creation of the ICD initial state can be monitored by detection of a photoelectron with a binding energy in the inner-valence range. (Core level ICD is not considered in this work.) We have shown earlier that photoelectron-ICD electron coincidence spectroscopy is a powerful technique to detect ICD of clusters larger than the dimer $[4,13,14]$. Details of the experiment reported here are as follows: Our cluster source uses expansion of Ne gas through a cryogenically cooled, conical nozzle made from copper [15]. The expansion pressure was set to 1.21 bar, the nozzle had a diameter of $80 \mu \mathrm{m}$, half an opening angle of $15^{\circ}$ and was cooled down to $48 \mathrm{~K}$. Applying an empirical scaling law [16], an expectation value for the cluster size of $\langle N\rangle=480$ follows. For further details of the apparatus see [14]. Electrons are detected by a highly efficient magnetic bottle spectrometer, the properties of which have been described [17]. Details of the design of this instrument can be found in [18]. Here, the most important property of the instrument is its high, and predictable detection efficiency of around $60 \%$ (see below). The experiment has been carried out at the undulator beamline UE112-PGM-1 of Helmholtz-Zentrum Berlin using the single bunch mode. In this mode of operation, synchrotron radiation arrives in flashes of few tens of ps length, with a separation in time of $\tau \approx 800$ ns. Using a small accelerating voltage into the drift tube of our spectrometer, the maximum flight time of electrons stayed below $\tau$. The decay time of ICD is in the fs range, and has no influence on the detection process. 


\section{Measured data and data analysis}

Figure 1 shows the electron, electron coincidence spectrum of Ne clusters. Each pixel of the color-coded map displays the number of electron pairs detected, with energy $e_{1}$ of the electron arriving first ('fast electron') plotted against the vertical axis, and energy $e_{2}$ of the second electron ('slow electron') plotted against the horizontal. A (small) background of random coincidences was determined by measuring the amount of $e_{1}$ electrons from the $n$th synchrotron radiation bunch arriving in coincidence with $e_{2}$ electrons from the $(n+1)$ th bunch, and was subtracted. Raw spectra recorded as timeof-flight maps were converted to kinetic energy using measured energies of atomic photolines. Data were recorded in list mode, and coincidence spectra were assembled by searching for events, in which two electrons were detected within the same bunch period.

For a given photon energy, the kinetic energy $e_{2 s}$ of photoelectrons pertaining to $2 s$ photoionization can be easily calculated (binding energy approx. $48.2 \mathrm{eV}$, see [5]). Inspecting the coincidence map in figure 1, the coincidences with ICD electrons of low kinetic energy $e_{I C D}$ can easily be found, in agreement with earlier results $[2,4]$. For unit efficiency of ICD, and recorded with an ideal detector, the intensity (events/time) of such coincidences would equal the intensity of primary $2 s$ photoelectrons, determined without discrimination for the occurence of a coincident partner electron. In a realistic experiment, already due to the not perfect solid angle acceptance of the spectrometer and the finite efficiency of its microchannel plate detector, for some of the primary photoelectrons the ICD electron is lost. We call the latter events 'singles', as opposed to 'doubles', in which two electrons were 
detected. The main idea laid out in this paper consists in a careful calibration of all apparative factors that may influence the singles/doubles ratio. Any deviation of this ratio from unity after correction for these factors would signal the presence of relaxation channels, which do not proceed via emission of an ICD electron.

We now formalize this idea: Let us write the count rate in the photoline (singles) as

$$
p\left(e_{p h}\right)=\gamma\left(e_{p h}\right) r_{p h}
$$

and the coincident count rate for photoelectron-autoionization electron coincidences (doubles) as

$$
P\left(e_{p h}, e_{a u}\right)=p\left(e_{p h}\right) \gamma\left(e_{a u}\right) \alpha_{a u}=\gamma\left(e_{p h}\right) r_{p h} \gamma\left(e_{a u}\right) \alpha_{a u}
$$

Here, $p$ is measured photoelectron count rate, independent of whether a second electron was coincident with the photoelectron or not, $P$ is the measured coincidence event rate, $\gamma$ is the detection probability (which might depend on kinetic energy $e_{p h}$ or $e_{a u}$, resp.), $r_{p h}$ is the rate of photoelectrons created (dependent on numerous factors that are difficult to quantify, such as photon flux, sample density, ionization cross section), $\alpha_{a u}$ is the summed branching ratio into all autoionization channels. This is the quantity to be determined. We use the fact that the MCP detector and the acquisition electronics are multi-hit capable (able to record several events within one period of length $\tau)$ as long as the electrons have some difference in kinetic energy.

From an experiment, $\alpha_{a u}$ can be determined as

$$
\frac{P\left(e_{p h}, e_{a u}\right)}{p\left(e_{p h}\right)} \frac{1}{\gamma\left(e_{a u}\right)}=\alpha_{a u} .
$$


$p, P$ are measured quantities, the apparative factor $\gamma\left(e_{a u}\right)$ remains to be determined.

The experimental value of $P$ can be determined from the data shown in 1 . The photoelectron data which lead to the value of the total count rate $p$ are shown in Fig. 2. Due to the worse energy resolution of the magnetic bottle spectrometer compared to an electrostatic analyser, the monomer component of the $2 s$ line, and the bulk-surface splitting within the cluster photoelectron line $[5,9]$ cannot be resolved. A flank at the high binding energy side of the peak is nevertheless visible in the total photoelectron signal, in particular when the peak shape is compared to the line profile derived from the coincident data (top panel of Fig. 1 or bottom trace in Fig. 2). Another reason why the monomer feature is less apparent than in earlier data [9] is a higher degree of condensation, which can be achieved with the expansion chamber layout used in the current experiment. The value of $p$ has been determined from the background-subtracted spectrum shown in Fig. 2 by integration between the vertical dashed lines.

The detection probability or transmission function $\gamma$ basically is the product of accepted solid angle (as a fraction of $4 \pi$ ) times probability to register a charged particle on the detector. It can be determined by comparing measured count rates for photoelectron, Auger electron coincidences from a rare gas. Here, we have used coincidences of Xe $4 d$ photoelectrons followed by Xe $N_{4,5} O O$ Auger decay, similar as described in [17]. The detection efficiency of our magnetic bottle has been measured for kinetic energies between 0 and $5 \mathrm{eV}$ and was found constant if the guiding magnetic field is chosen not too low [17]. We therefore neglect an energy dependence of $\gamma$. 
Table 1: Results of the experiment. The error of the ICD efficiency is calculated using error propagation assuming a $5 \%$ uncertainty in the detection efficiency and a $5 \%$ error in $p$ due to non-perfect separation against atomic photoelectrons.

\begin{tabular}{cccc}
\hline \hline all events & coinc. events & detection efficiency & ICD efficiency \\
$p * 30 \mathrm{~s}$ & $P * 30 \mathrm{~s}$ & $\gamma$ & $\alpha_{a u}$ \\
\hline 397881 & 244091 & $0.62 \pm 0.05$ & $0.99 \pm 0.11$ \\
\hline
\end{tabular}

\section{Results and Discussion}

The main result of our study is given in Tab. 1. Experimentally, we find unit efficiency of the ICD process in $\langle N\rangle=480$ Ne clusters within the sensitivity limit of our experiment. This is in line with an earlier experiment [9].

We have so far discarded the influence of inelastic scattering. In the current data set, this process seems to be unimportant. This can be explained with the low kinetic energy of both photoelectron and ICD electron. In [9], measured at $60.5 \mathrm{eV}$, the results suggested a few \% intensity loss from the photoelectron line. Coincident data measured over a range of photon energies exist and will be presented in a forthcoming publication.

In summary, we have presented a case study of the efficiency of Interatomic Coulombic Decay by a method, which will be applicable to numerous systems. While our earlier work on the same topic [9] relied on the separate, spectroscopic identification of the photoelectron and the ICD electron peak, the method presented here directly measures the correlated electron pairs. We therefore expect that it will still be useable when the identification of the 
respective peaks in a conventional electron spectrum is difficult because of congestion by scattered electrons, or overlapping signal from monomers.

\section{Acknowledgements}

This work has been partially funded by the Deutsche Forschungsgemeinschaft (FOR 1789). We thank HZB for the allocation of synchrotron radiation beamtime. 


\section{References}

[1] L. S. Cederbaum, J. Zobeley, F. Tarantelli, Phys. Rev. Lett. 79 (1997) 4778-4781.

[2] S. Marburger, O. Kugeler, U. Hergenhahn, T. Möller, Phys. Rev. Lett. 90 (2003) 203401.

[3] V. Averbukh, P. Demekhin, P. Kolorenc, S. Scheit, S. D. Stoychev, A. I. Kuleff, Y.-C. Chiang, K. Gokhberg, S. Kopelke, N. Sisourat, L. S. Cederbaum, Journal of Electron Spectroscopy and Related Phenomena Electron Spectroscopy Kai Siegbahn Memorial Volume 183 (2011) 36.

[4] U. Hergenhahn, J. Electron Spectrosc. Relat. Phenom. 184 (2011) 78.

[5] G. Öhrwall, M. Tchaplyguine, M. Lundwall, R. Feifel, H. Bergersen, T. Rander, A. Lindblad, J. Schulz, S. Peredkov, S. Barth, S. Marburger, U. Hergenhahn, S. Svensson, O. Björneholm, Phys. Rev. Lett. 93 (2004) 173401.

[6] F. Trinter, J. B. Williams, M. Weller, M. Waitz, M. Pitzer, J. Voigtsberger, C. Schober, G. Kastirke, C. Müller, C. Goihl, P. Burzynski, F. Wiegandt, T. Bauer, R. Wallauer, H. Sann, A. Kalinin, L. P. H. Schmidt, M. Schöffler, N. Sisourat, T. Jahnke, Phys. Rev. Lett. 111 (2013) 093401.

[7] K. Schnorr, A. Senftleben, M. Kurka, A. Rudenko, L. Foucar, G. Schmid, A. Broska, T. Pfeifer, K. Meyer, D. Anielski, R. Boll, D. Rolles, M. Kübel, M. F. Kling, Y. H. Jiang, S. Mondal, T. Tachibana, K. Ueda, 
T. Marchenko, M. Simon, G. Brenner, R. Treusch, S. Scheit, V. Averbukh, J. Ullrich, C. D. Schröter, R. Moshammer, Phys. Rev. Lett. 111 (2013) 093402.

[8] Note. This remark is somewhat hypothetical for the Ne case, as at least in the dimer the nuclear dynamics is well understood. Generally however, systems can be anticipated in which ultrafast nuclear conversion already may gain energy in the intermediate state, in competition with ICD.

[9] S. Barth, S. Marburger, O. Kugeler, V. Ulrich, S. Joshi, A. M. Bradshaw, U. Hergenhahn, Chem. Phys. 329 (2006) 246-250.

[10] R. Dörner, V. Mergel, O. Jagutzki, L. Spielberger, J. Ullrich, R. Moshammer, H. Schmidt-Böcking, Phys. Rep. 330 (2000) 95-192.

[11] T. Jahnke, A. Czasch, M. S. Schöffler, S. Schössler, A. Knapp, M. Käsz, J. Titze, C. Wimmer, K. Kreidi, R. E. Grisenti, A. Staudte, O. Jagutzki, U. Hergenhahn, H. Schmidt-Böcking, R. Dörner, Phys. Rev. Lett. 93 (2004) 163401.

[12] S. Thürmer, M. Oncak, N. Ottosson, R. Seidel, U. Hergenhahn, S. E. Bradforth, P. Slavíček, B. Winter, Nature Chemistry 5 (2013) 590.

[13] M. Mucke, M. Braune, S. Barth, M. Förstel, T. Lischke, V. Ulrich, T. Arion, U. Becker, A. Bradshaw, U. Hergenhahn, Nature Physics 6 (2010) 143-146.

[14] T. Arion, M. Mucke, M. Förstel, A. M. Bradshaw, U. Hergenhahn, J. Chem. Phys. 134 (2011) 74306. 
214

215

216

217

218

219

220

221

[15] S. P. Marburger, O. Kugeler, U. Hergenhahn, AIP Conference Proceedings 705 (2004) 1114-1117.

[16] O. F. Hagena, Rev. Sci. Instrum. 63 (1992) 2374-2379.

[17] M. Mucke, M. Förstel, T. Lischke, T. Arion, A. M. Bradshaw, U. Hergenhahn, Rev. Sci. Instrum. 83 (2012) 063106.

[18] M. Mucke, Employing electron-electron coincidence techniques to investigate the autoionisation of clusters, Ph.D. thesis, Technical University Berlin, 2011. 


\section{Figure Captions}

Figure 1: Electron-electron coincidence spectrum of Ne clusters recorded with a photon energy of $h \nu=52 \mathrm{eV}$. Panel a) Energy distribution of the 'fast' electron on a binding energy scale (horizontal axis). Panel b) Colour coded map of the number of events, in which a fast electron with kinetic energy $h \nu-e_{1}$ (horizontal axis) and a slow electron with energy $e_{2}$ (vertical axis) have been detected. Events in b) are per pixel of $100 \times 100 \mathrm{meV}$ size, the colour scale is linear. Panel a) is derived from b) by summation of all events along lines of constant $e_{1}$. By their binding energy, the fast electrons can be identified as Ne $2 s$ photoelectrons. The energy distribution of slow electrons agrees with earlier measurements of Ne ICD in larger clusters [9]. A background of inelastically scattered electrons is shown in panel a).

Figure 2: Ne $2 s$ photoelectron spectrum of Ne clusters recorded with a photon energy of $h \nu=52 \mathrm{eV}$, vs. binding energy $e_{1}$. Black, solid trace: All events (sum of singles and doubles). Red trace: Background of inelastically scattered electrons. Upper dashed trace (blue): All events, background subtracted. Lower dashed trace (green): Coincident events only. The latter is identical to the green, dashed spectrum in panel a) of Fig. 1. Intensity is expressed as events/eV. 


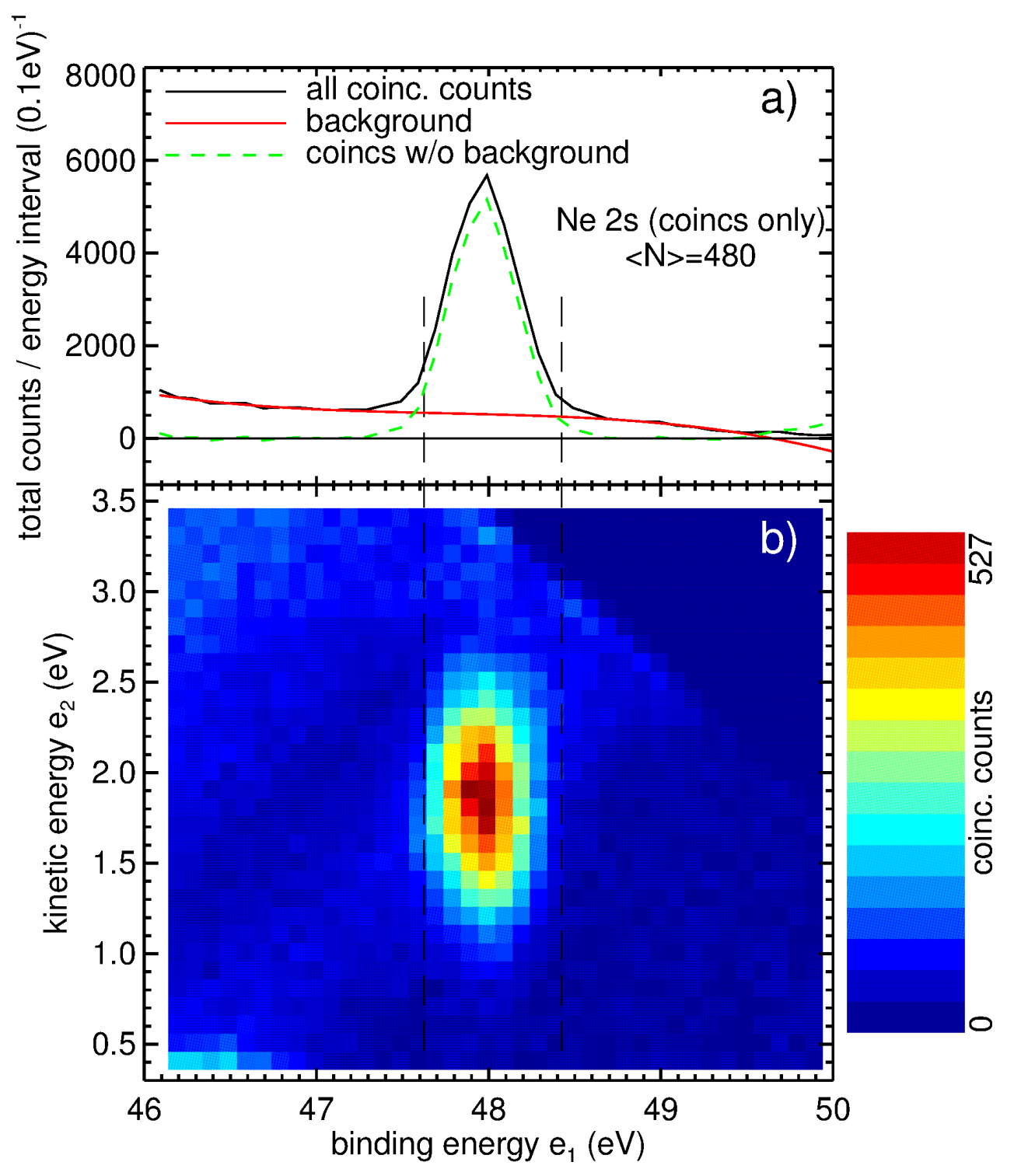

Figure 1: 


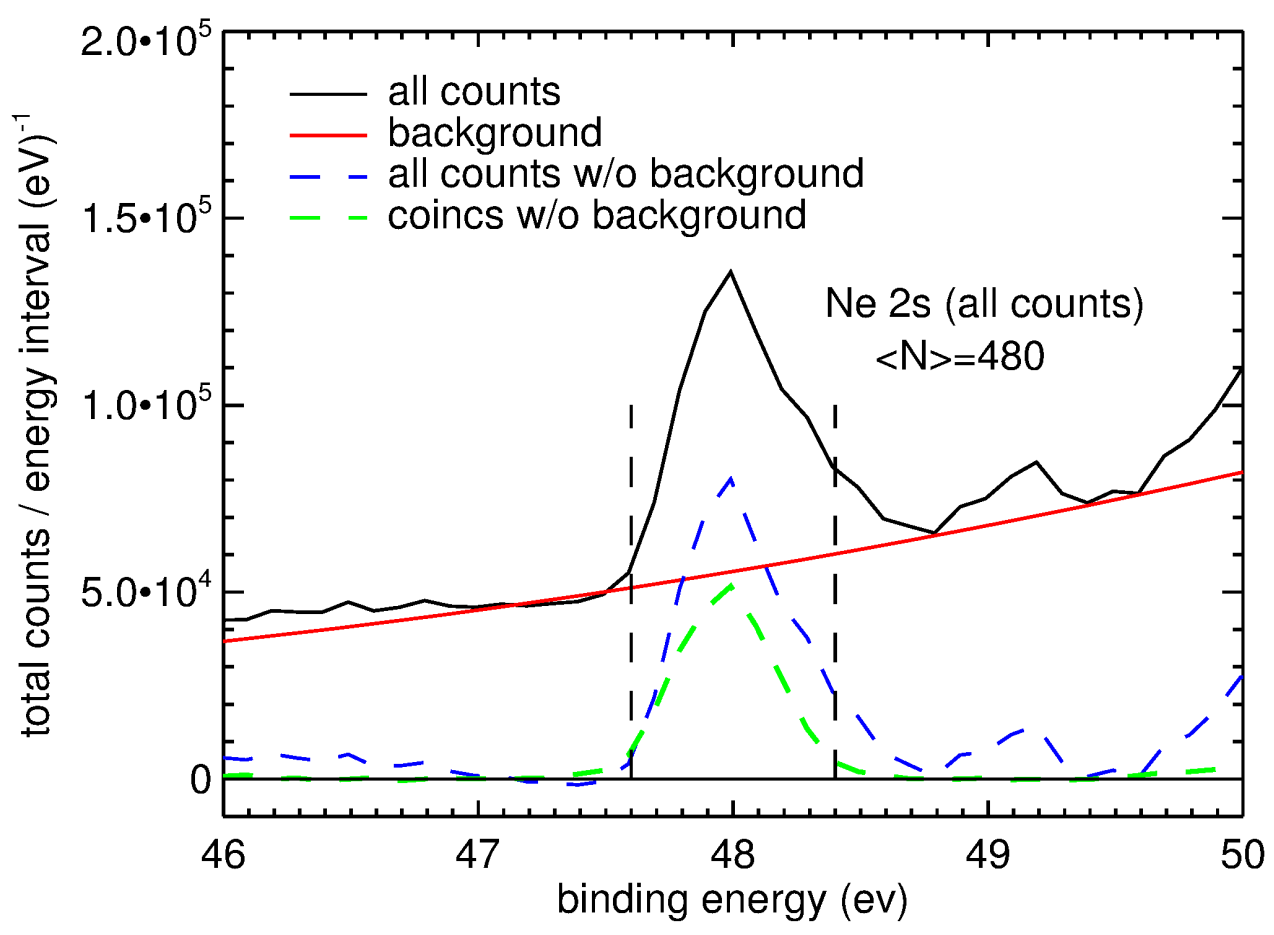

Figure 2: 